\title{
ARTICLE \\ Maintenance of antidepressant and antisuicidal effects by D-cycloserine among patients with treatment-resistant depression who responded to low-dose ketamine infusion: a double-blind randomized placebo-control study
}

\author{
Mu-Hong Chen (DD $^{1,2,3}$, Chih-Ming Cheng (D) $^{1,2,3,4}$, Ralitza Gueorguieva ${ }^{5,6}$, Wei-Chen Lin ${ }^{1,2,3}$, Cheng-Ta Li ${ }^{1,2,3}$, Chen-Jee Hong ${ }^{1,2,3}$, \\ Pei-Chi Tu ${ }^{1,2,7}$, Ya-Mei Bai ${ }^{1,2,3}$, Shih-Jen Tsai $\mathbb{i D}^{1,2,3}$, John H. Krystal ${ }^{5,8,9,10}$ and Tung-Ping Su ${ }^{1,2,3,7,11}$
}

Increasing evidence supports a rapid antidepressant and antisuicidal effect of a single subanesthetic dose of ketamine infusion for treatment-resistant depression (TRD). Maintaining the initial clinical response after ketamine infusion in TRD is a crucial next-step challenge. D-cycloserine (DCS), a partial agonist of the glycine co-agonist of the N-methyl-D-aspartate (NMDA) glutamate receptor, is potentially effective as a depression augmentation treatment. However, whether DCS maintains the antidepressant and antisuicidal effects of ketamine infusion remains unknown. In all, 32 patients with TRD (17 with major depression and 15 with bipolar depression) who responded to ketamine infusion with an average 17-item Hamilton Depression Rating Scale (HAMD) score of $9.47 \pm 4.11$ at baseline were randomly divided to 6-week DCS treatment ( $250 \mathrm{mg}$ for 2 days, $500 \mathrm{mg}$ for 2 days, $750 \mathrm{mg}$ for 3 days, and $1000 \mathrm{mg}$ for 5 weeks) and placebo groups. Depression symptoms were rated at timepoints of dose titration and weekly. During the 6-week treatment, the total scores of HAMD did not differ between the DCS and placebo groups. The results remained consistent when stratified by disorder. A mixed model analysis indicated that the DCS group exhibited lower scores of HAMD item 3 (suicide) compared with the placebo group throughout the follow-up period $(p=0.01)$. A superior maintenance of the antisuicidal effect of ketamine was observed in the DCS group than in the placebo group. DCS may be therapeutically beneficial for patients with TRD who responded to ketamine infusion but have a residual suicidal risk.

Neuropsychopharmacology (2019) 44:2112-2118; https://doi.org/10.1038/s41386-019-0480-y

\section{INTRODUCTION}

The Sequenced Treatment Alternatives to Relieve Depression study reported that up to $40 \%$ of the patients with major depressive disorders did not achieve symptomatic remission after two trials of traditional antidepressants, which is defined as treatment-resistant major depression [1]. In comparison with treatment-resistant major depression, treatment-resistant bipolar depression has been less studied, although depressive and dysthymic episodes contribute to the majority of the clinical course of bipolar disorder [2-4]. Treatment resistance in major depression and bipolar depression has been commonly coupled with functional impairment, poor quality of life, suicide ideation and attempts, self-injurious behaviors, and a high relapse rate $[2,4,5]$.

Increasing evidence suggests a rapid antidepressant effect of a single subanesthetic dose of ketamine infusion for treatmentresistant major depression and bipolar depression [6-8]. Beyond the rapid antidepressant effect of ketamine infusion, a recent meta-analysis supports a rapid antisuicidal effect of a single lowdose ketamine infusion in patients with treatment-resistant major depression and bipolar depression [9]. Wilkinson et al. indicated that ketamine rapidly reduced suicidal thoughts within 1 day and for up to 1 week in depressed patients with suicidal ideation and further determined that ketamine's effects on suicidal ideation were partially independent of its effects on depression [9]. Thus, the underlying mechanisms of ketamine effects on depression and suicidality mitigation may differ.

However, maintaining the initial response of ketamine infusion is an important therapeutic challenge in the psychiatric clinical practice [10]. The benefits of ketamine may be extended by repeated dosing, but ketamine infusion is resource intensive and is associated with risks, including addiction liability, dissociative symptoms, and nausea [11]. Finding an optimal drug that can maintain the antidepressant effects of ketamine infusion may be clinically beneficial for patients with treatment-resistant major depression and bipolar depression [10].

${ }^{1}$ Division of Psychiatry, Faculty of Medicine, National Yang-Ming University, Taipei, Taiwan; ${ }^{2}$ Department of Psychiatry, Taipei Veterans General Hospital, Taipei, Taiwan; ${ }^{3}$ Institute of Brain Science, National Yang-Ming University, Taipei, Taiwan; ${ }^{4}$ Department of Psychiatry, Yuanshan branch, Taipei Veterans General Hospital, Yilan, Taiwan; ${ }^{5}$ Department of Psychiatry, Yale University School of Medicine, New Haven, CT, USA; ${ }^{6}$ Department of Biostatistics, School of Public Health, Yale University, New Haven, CT, USA; ${ }^{7}$ Department of Medical Research, Taipei Veterans General Hospital, Taipei, Taiwan; ${ }^{8}$ Department of Neuroscience, Yale University School of Medicine, New Haven, CT, USA; ${ }^{9}$ Yale-New Haven Hospital, New Haven, CT, USA; ${ }^{10}$ VA National Center for PTSD, VA Connecticut Healthcare System, West Haven, CT, USA and ${ }^{11}$ Department of Psychiatry, Cheng Hsin General Hospital, Taipei, Taiwan

Correspondence: John H. Krystal (john.krystal@yale.edu) or Tung-Ping Su (tomsu0402@gmail.com)

Received: 21 March 2019 Revised: 1 August 2019 Accepted: 7 August 2019

Published online: 17 August 2019 
D-cycloserine (DCS), a partial agonist of the glycine site of NMDA glutamate receptors, has been reported to be potentially effective for depression augmentation treatment $[12,13]$. Heresco-Levy et al. reported that only high doses ( $1000 \mathrm{mg} /$ day) of DCS, which act as a functional NMDAR antagonist, but not low doses ( $250 \mathrm{mg} /$ day), are significantly effective for treatment-resistant depression (TRD) $[12,14]$. A small sample size $(n=7)$ open-label study suggested a potential role of DCS in the maintenance of the antidepressant effect of a single dose of ketamine infusion among patients with treatmentresistant bipolar depression [15]. However, whether DCS can really maintain the antidepressant effect of ketamine infusion in a randomized placebo-control study design setting remains unknown.

In this study, we conducted a two-phase clinical trial composed of a ketamine infusion open-label trial (phase 1 study) and a doubleblind randomized DCS-placebo control trial (phase 2 study). Patients with treatment-resistant major depression or bipolar depression who responded to ketamine infusion in the phase 1 study were enrolled in the phase 2 study for the investigation of the role of DCS in the maintenance of the initial response of ketamine. We hypothesized that DCS may be superior to a placebo in maintaining the antidepressant effect of ketamine infusion.

\section{METHODS}

Inclusion criteria of patients

We enrolled 49 patients aged between 21 and 65 years with a Diagnostic and Statistical Manual of Mental Disorders, 4th Edition, Text Revision diagnosis of major depressive disorder or bipolar disorder or major depressive episode. In addition, they met the criteria of TRD (treatment-resistant major depression and bipolar depression). Treatment-resistant major depression was defined as the failure of treatment response for at least two different antidepressants with adequate dosage and treatment duration [7].

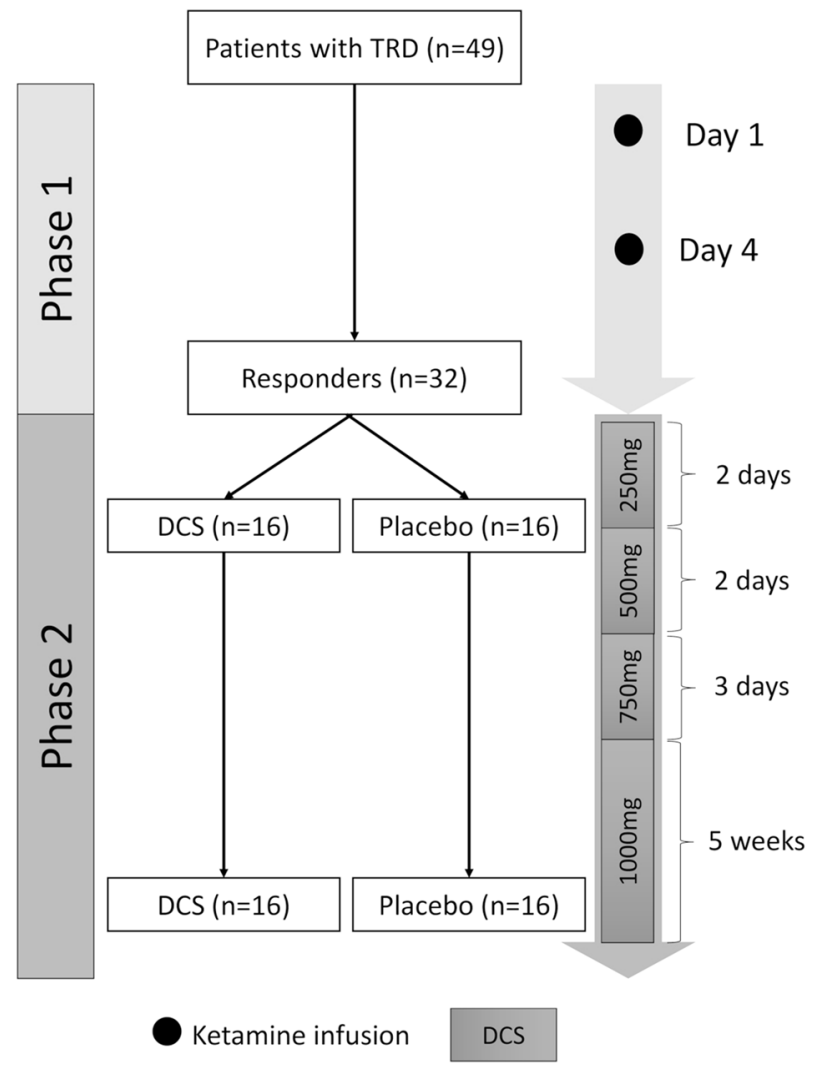

Fig. 1 Study design flow chart. TRD treatment-resistant depression (major depression, bipolar depression), DCS D-cycloserine
Treatment-resistant bipolar depression was defined as a lack of response to at least two trials with antidepressants or mood stabilizers with documented efficacy in bipolar depression (lithium, lamotrigine, quetiapine, or olanzapine) in adequate doses and treatment duration [16]. Mini-International Neuropsychiatric Interview (MINI) for depression and suicidality and Maudsley staging method (MSM) for level of treatment resistance were also assessed $[17,18]$. Patients were excluded if they had a history of any major medical or neurological illness (i.e., stroke or seizure), alcohol or substance abuse, and mild symptoms (17-item Hamilton Depression Rating Scale (HAMD) score $<16$ before study entry) [19]. This clinical trial was registered in the UMIN Clinical Trials Registry (R000027142-UMIN000023581). This study was performed in accordance with the Declaration of Helsinki, and was approved by the Taipei Veterans General Hospital Institutional Review Board. Informed consent was provided by all participants.

\section{Study procedures}

This study was composed of phase 1 open-label ketamine infusion study and phase 2 double-blind randomized DCS-placebo control study (Fig. 1). In the phase 1 study, patients with TRD received the add-on 40 -min intravenous ketamine $(0.5 \mathrm{mg} / \mathrm{kg})$ infusions at day 1 and day 4 of the phase 1 study. Depressive symptoms were rated using the HAMD on day 1 (first ketamine infusion: $0,40,80,120$, and $240 \mathrm{~min}$ ), day 2 , day 4 (second ketamine infusion: $0,40,80,120$, and $240 \mathrm{~min}$ ), day 5 , and day 7 . The purpose of two ketamine infusions is to improve the response rate in the phase 1 study, because our previous clinical trial study found only $\sim 50 \%$ of the response rate after a single dose $(0.5 \mathrm{mg} / \mathrm{kg})$ of ketamine infusion in Taiwanese patients with TRD [7]. Responder status was defined by the response

Table 1. Demographic and clinical characteristic among patients with treatment-resistant depression in phase 1 open-label study

\begin{tabular}{|c|c|c|c|c|}
\hline & $\begin{array}{l}\text { All patients } \\
(n=49)\end{array}$ & Bipolar $(n=19)$ & $\operatorname{MDD}(n=30)$ & $p$-value \\
\hline Age (years, SD) & $47.35(10.66)$ & $45.47(11.33)$ & $48.53(10.23)$ & 0.33 \\
\hline Male $(n, \%)$ & $13(26.5 \%)$ & $5(26.3 \%)$ & $8(26.7 \%)$ & $>0.99$ \\
\hline Education (years, SD) & $13.47(2.79)$ & $13.68(2.63)$ & $13.33(2.93)$ & 0.67 \\
\hline $\begin{array}{l}\text { Duration of illness } \\
\text { (years, SD) }\end{array}$ & $13.69(8.43)$ & $14.74(6.94)$ & $13.03(9.30)$ & 0.50 \\
\hline \multicolumn{5}{|c|}{ Psychiatric comorbidities } \\
\hline PD & 16 (32.7\%) & 7 (36.8\%) & $9(30.0)$ & 0.62 \\
\hline PTSD & $2(4.1 \%)$ & $0(0.0 \%)$ & $2(6.7 \%)$ & 0.52 \\
\hline GAD & $21(42.9 \%)$ & $6(31.6 \%)$ & 15 (50.0\%) & 0.25 \\
\hline Suicide history $(n, \%)$ & $24(49.0 \%)$ & 11 (57.9\%) & $13(43.3 \%)$ & 0.39 \\
\hline \multicolumn{5}{|l|}{ HAMD score (SD) } \\
\hline Baseline (day 1) & $22.08(3.54)$ & $22.42(3.25)$ & $21.87(3.75)$ & 0.60 \\
\hline \multicolumn{5}{|l|}{ First injection (day 1) } \\
\hline $40 \mathrm{~min}$ & $14.67(5.61)$ & $14.47(4.98)$ & $14.80(6.05)$ & 0.85 \\
\hline $80 \mathrm{~min}$ & $13.90(5.59)$ & $13.63(5.42)$ & $14.07(5.78)$ & 0.79 \\
\hline $120 \mathrm{~min}$ & $13.27(5.40)$ & $13.16(5.74)$ & $13.33(5.64)$ & 0.91 \\
\hline $240 \mathrm{~min}$ & $13.06(5.40)$ & $13.16(5.74)$ & $13.00(5.28)$ & 0.92 \\
\hline Day 2 & $12.90(6.21)$ & $12.79(6.92)$ & $12.97(5.85)$ & 0.92 \\
\hline $\begin{array}{l}\text { Second injection } \\
\text { (day 4) }\end{array}$ & $13.96(6.60)$ & $14.53(7.91)$ & $13.60(5.73)$ & 0.64 \\
\hline $40 \mathrm{~min}$ & $11.98(6.41)$ & $12.37(7.02)$ & $11.73(6.10)$ & 0.74 \\
\hline $80 \mathrm{~min}$ & $11.84(6.54)$ & $12.63(7.51)$ & $11.33(5.93)$ & 0.50 \\
\hline $120 \mathrm{~min}$ & $11.80(6.60)$ & $12.53(7.73)$ & $11.33(5.88)$ & 0.54 \\
\hline $240 \mathrm{~min}$ & $11.78(6.53)$ & $12.16(7.37)$ & $11.53(6.05)$ & 0.75 \\
\hline Day 5 & $12.33(6.93)$ & $12.63(8.49)$ & $12.13(5.89)$ & 0.81 \\
\hline Day 7 & $13.20(7.02)$ & $13.63(8.25)$ & $12.93(6.25)$ & 0.74 \\
\hline Response rate $(n, \%)$ & $32(65 \%)$ & $13(68.4 \%)$ & 19 (63.3\%) & 0.77 \\
\hline
\end{tabular}

HAMD Hamilton Depression Rating Scale, PD panic disorder, PTSD posttraumatic stress disorder, GAD generalized anxiety disorder, $S D$ standard deviation 
( $\geq 50 \%$ reduction of mood ratings) at any two daily HAMD measures during the period of day 1 to day 7 post-ketamine administration. Only those who responded to ketamine infusion $(n=32)$ were enrolled in the phase 2 double-blind randomized DCS-placebo control study. In the phase 2 study, patients with TRD were randomly divided into 6-week DCS (Seromycin, Eli Lilly) treatment and placebo groups. For the DCS group, the dose titration procedure was applied from $250 \mathrm{mg} /$ day for 2 days, $500 \mathrm{mg} /$ day for 2 days, and $750 \mathrm{mg} /$ day for 3 days to $1000 \mathrm{mg} /$ day for 5 weeks. The final dose was adjusted in the range of $500-1000 \mathrm{mg} /$ day based on the patients' tolerability. Depression symptoms were rated at timepoints of dose titration and weekly.

\section{Statistical analysis}

The data were checked for normality prior to analysis. There were no missing data in our study. Independent $t$ tests for continuous variables and Fisher's chi-square tests for nominal variables were used to assess between-group differences in baseline demographic and clinical data. In the phase 1 study, a mixed model analysis with an autoregressive correlation structure for the repeated measures on the same individual over time was used to assess the effects of ketamine on depression symptoms during the treatment period with group (major depression vs. bipolar depression) as a betweenpatient factor, time (day 1 to day 7) as a within-patient factor, and baseline depression symptoms as a between-patient predictor, as well as all possible interactions. In the phase 2 study, mixed model analyses with autoregressive correlation structure of the repeated measures for the same individual over time was used to assess the effects of DCS on the total HAMD scores and on item 3 (suicide)
HAMD scores during the treatment period with group (DCS vs. placebo) as a between-patient factor, time (week 1 to week 6) as a within-patient factor, and baseline depression (after ketamine infusion) scores as a between-patient predictor, as well as all possible interactions. Furthermore, separate analyses for patients with major depression and with bipolar depression were also conducted to investigate the potential role of disorder type in DCS treatment efficacy. A two-tailed $p$-value $<0.05$ was considered statistically significant. All data processing and statistical analyses were performed using the SPSS version 21 software (SPSS Inc.).

\section{RESULTS}

Phase 1 open-label ketamine infusion study

In the phase 1 study, 30 patients with treatment-resistant major depression and 19 with treatment-resistant bipolar depression were enrolled, with an average age of $47.35(S D=10.66)$ years and predominantly female (73.5\%; Table 1). Average HAMD scores were $22.08(S D=3.54)$ at baseline before ketamine infusion. The response rate was $65 \%(n=32)$ after two ketamine infusions, and did not differ between major depression and bipolar depression (63.3\% vs. $68.4 \%, p=0.77)$. The mixed model analyses demonstrated that the repeated ketamine infusions were effective for treatment-resistant major depression $(F(1,65)=17.58, p<0.001)$ and bipolar depression $(F(1,27)=10.24, p=0.003)$ (Fig. 2).

Phase 2 double-blind randomized DCS-placebo control study In phase 2 double-blind randomized placebo control study, 32 patients with TRD who responded to ketamine infusions in the

a)

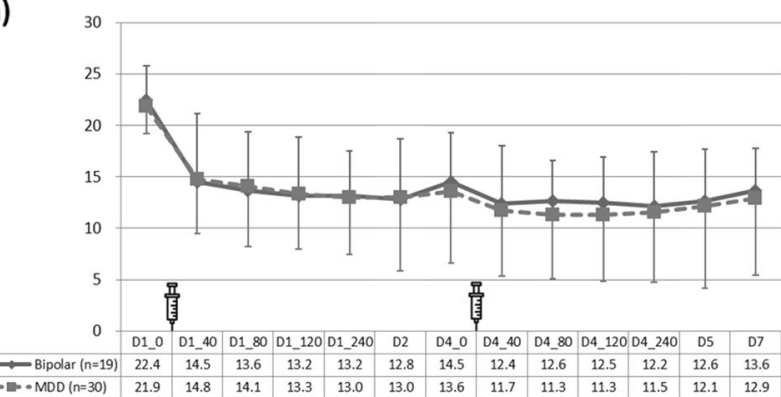

b)

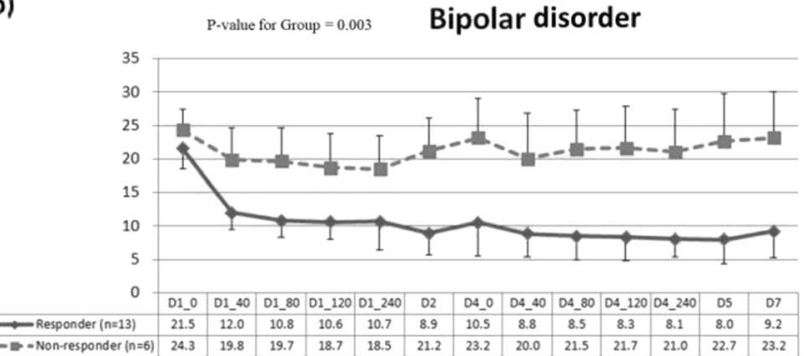

d)

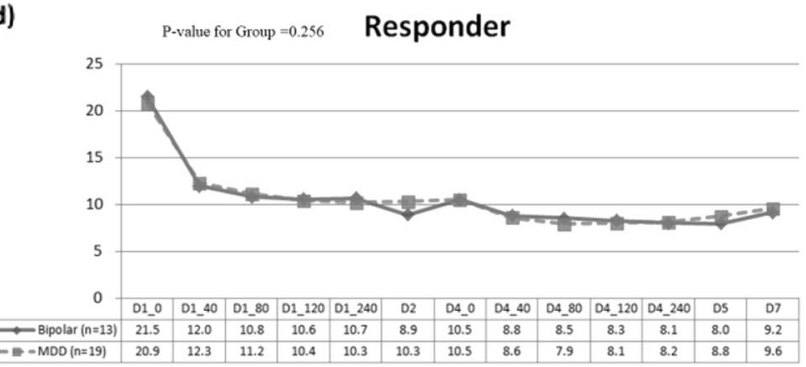

c)

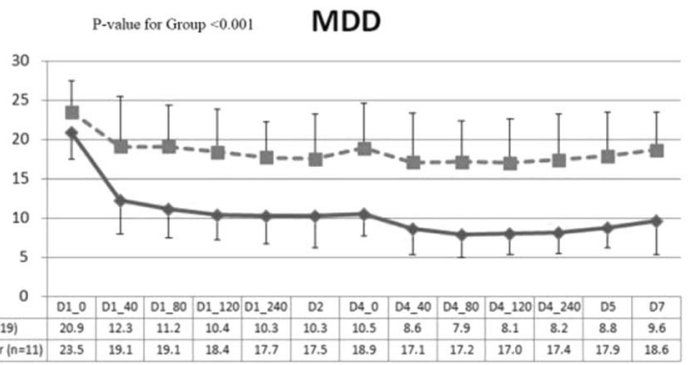

e)

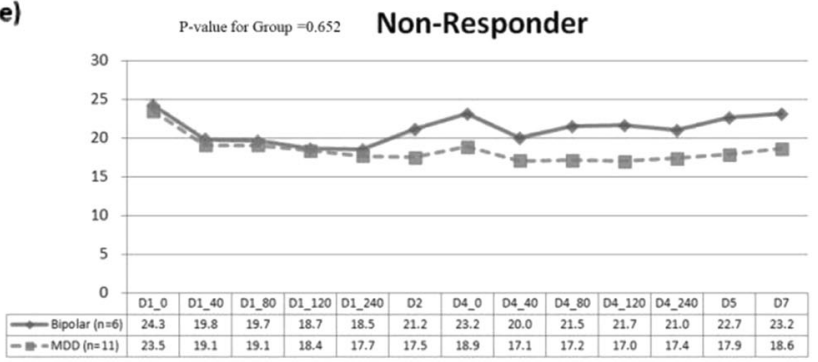

Fig. 2 Trajectory of depression in phase 1 open-label study (the mixed model with adjustment of baseline score, age and sex). a Total patients, b bipolar disorder patients, c major depressive disorder (MDD) patients, $\mathbf{d}$ responders, and e non-responders 
phase 1 study were enrolled. With the 6-week DCS or placebo treatment, the total HAMD scores did not differ throughout the follow-up period between the DCS and placebo groups $(F(1,27)=$ 1.11, $p=0.30$; Table 2 and Fig. 3). The interaction between disorder type and treatment was nonsignificant $(F(1,24)=1.93$, $p=0.18)$. Separate analyses for individuals with major depression $(F(1,31)=0.09, p=0.77)$ and with bipolar depression $(F(1,33)=$ 2.26, $p=0.14$ ) demonstrated that the maintenance of the antidepressant effect of ketamine infusion was similar between the DCS and placebo groups (Fig. 3).

Specifically analyzing the role of DCS in the maintenance of antisuicidal effect, measured by item 3 (suicide) of HAMD, of ketamine infusion, we found that the DCS group exhibited lower scores of HAMD item 3 than the placebo group throughout the follow-up period $(\mathrm{F}(1,26)=7.91, p=0.01$; Fig. 4).

Adverse effects, including dizziness $(18.8 \%$ vs. $6.3 \%, p=0.600)$, sedation $(18.8 \%$ vs. $0 \%, p=0.226)$, hand tremor $(12.5 \%$ vs. $0 \%$, $p=0.484)$, and itching ( $6.3 \%$ vs. $0 \%, p>0.999)$, were rare and did

Table 2. Demographic and clinical characteristic among patients with treatment-resistant depression in phase 2 double-blind randomized placebo-control study

\begin{tabular}{|c|c|c|c|}
\hline & $\begin{array}{l}\text { DCS group } \\
(n=16)\end{array}$ & $\begin{array}{l}\text { Control group } \\
(n=16)\end{array}$ & $p$-value \\
\hline Age (years, SD) & $43.50(11.00)$ & $48.81(9.70)$ & 0.16 \\
\hline Male $(n, \%)$ & $5(31.3 \%)$ & $5(31.3 \%)$ & $>0.99$ \\
\hline Diagnosis ( $n, \%)$ & & & $>0.99$ \\
\hline Major depression & $10(62.5 \%)$ & 7 (43.8\%) & \\
\hline Bipolar depression & $6(37.5 \%)$ & $9(56.3 \%)$ & \\
\hline Education (years, SD) & $13.94(2.79)$ & $13.88(3.12)$ & 0.95 \\
\hline $\begin{array}{l}\text { Duration of illness } \\
\text { (years, SD) }\end{array}$ & $13.56(10.53)$ & $12.69(5.63)$ & 0.77 \\
\hline \multicolumn{4}{|c|}{ Psychiatric comorbidities } \\
\hline PD & $4(25.0 \%)$ & $6(37.5 \%)$ & 0.70 \\
\hline PTSD & $1(6.3 \%)$ & $0(0.0 \%)$ & $>0.99$ \\
\hline GAD & $5(31.3 \%)$ & 7 (43.8\%) & 0.72 \\
\hline Suicide history $(n, \%)$ & $7(43.8 \%)$ & $8(50.0 \%)$ & $>0.99$ \\
\hline \multicolumn{4}{|l|}{ HAMD score (SD) } \\
\hline Baseline (day 1) & $9.75(3.26)$ & $9.19(4.92)$ & 0.71 \\
\hline Day 4 & $9.06(3.11)$ & $8.75(5.08)$ & 0.84 \\
\hline Day 7 & $11.06(5.09)$ & $9.25(5.41)$ & 0.34 \\
\hline Day 14 & $11.19(5.34)$ & $10.13(4.92)$ & 0.56 \\
\hline Day 21 & $11.31(5.77)$ & $11.50(6.21)$ & 0.93 \\
\hline Day 28 & $11.56(4.24)$ & $9.81(5.60)$ & 0.33 \\
\hline Day 35 & $11.13(4.77)$ & $10.06(6.46)$ & 0.60 \\
\hline Day 42 & $10.94(4.68)$ & $10.31(6.58)$ & 0.76 \\
\hline \multicolumn{4}{|c|}{ HAMD item 3 score (SD) } \\
\hline Baseline (day 1) & $0.31(0.48)$ & $0.19(0.54)$ & 0.495 \\
\hline Day 4 & $0.25(0.45)$ & $0.25(0.58)$ & 0.183 \\
\hline Day 7 & $0.50(0.73)$ & $0.38(0.62)$ & 0.239 \\
\hline Day 14 & $0.44(0.73)$ & $0.38(0.62)$ & 0.239 \\
\hline Day 21 & $0.50(0.73)$ & $0.56(0.73)$ & 0.258 \\
\hline Day 28 & $0.44(0.51)$ & $0.31(0.60)$ & 0.198 \\
\hline Day 35 & $0.38(0.62)$ & $0.38(0.62)$ & 0.219 \\
\hline Day 42 & $0.44(0.63)$ & $0.38(0.62)$ & 0.221 \\
\hline
\end{tabular}

not differ between DCS and placebo groups (Supplementary Table 1).

\section{DISCUSSION}

Our phase 2 randomized double-blind placebo control study findings did not support the study hypothesis that DCS augmentation treatment was superior to placebo in maintaining the initial antidepressant response to ketamine infusion. However, DCS did appear to maintain the antisuicidal effect of ketamine infusion during the 6-week follow-up period compared with the placebo. In addition, our phase 1 study findings revealed that the two infusions of ketamine per week improved the overall treatment response, up to $65 \%$, among Taiwanese patients with TRD.

One question raised by the current findings is whether DCS may play a role in depression treatment by enhancing or inhibiting NMDA receptor function. Ketamine is an NMDA receptor antagonist, but it enhances neuroplasticity with intermittent administration [20]. DCS is a weak partial agonist at GluN2Aand GluN2B-containing NMDA receptors, with greater agonist efficacy at GluN2C and GluN2D-containing receptors [21, 22]. Thus DCS may preferentially facilitate or inhibit some NMDA receptor subtypes. The failure of DCS to attenuate depression in this study may suggest that DCS is not having beneficial effects in patients by blocking NMDA receptors, i.e., it is not simply a "weaker ketamine." Instead, DCS may work by enhancing NMDA receptor function. Animal studies showed that DCS facilitated hippocampal long-term synaptic plasticity via the enhanced magnitude of NMDA receptor-dependent long-term depression and the decreased neurotransmission mediated by alpha-amino-3hydroxy-5-methyl-4-isoxazolepropionic acid/kainate subtypes of glutamate receptors $[23,24]$. Gupta et al. facilitated fear extinction via the increased activation of the MAPK signaling pathway in the orbitofrontal cortex and the increased pERK levels in the infralimbic prefrontal cortex, prelimbic prefrontal cortex intercalated cells, and lateral nucleus of the central amygdala $[25,26]$. Furthermore, as mentioned in the Introduction section, only the high-dose, but not low-dose, administration of DCS was effective for depression [12, 14]. Previous studies also revealed that lowdose DCS failed to enhance executive cognitive function in neurologically intact adults or in adults with stroke $[27,28]$. In this way, we suggested that high-dose DCS might attenuate executive cognitive deficits associated with suicide risk [29] or promoting the impact of anti-suicide psychosocial treatments [30, 31]. However, executive cognitive function was not assessed in this study. Further studies would be necessary to clarify the association between DCS, cognitive function, and suicidality.

Differences between the current study sample and the earlier study may have contributed to the failure to replicate the antidepressant efficacy of DCS. The earlier study excluded patients with significant suicide risk [12], while in this study nearly half of the patients had attempted suicide and approximately onequarter of patients had moderate-to-high risk based on the MINI. It is also possible that patients in this study had more severely treatment-resistant symptoms, with over two-thirds of patients meeting criteria for moderate-to-high treatment resistance based on MSM $[17,18]$.

A second problem may have been the confounding effect of persisting antidepressant effects of ketamine during the trial. The findings from the mixed samples of previous ketamine infusion studies for TRD patients with and without concomitant medications showed that $\sim 80 \%$ of patients with TRD may relapse within 18 days or so after a single ketamine infusion $[6,32]$. However, the duration of relapse between add-on and drug-free studies remained unknown. Increasing evidence reported that repeated injections may prolong the antidepressant effect of ketamine infusion [33-35]. Our previous studies demonstrated that the 


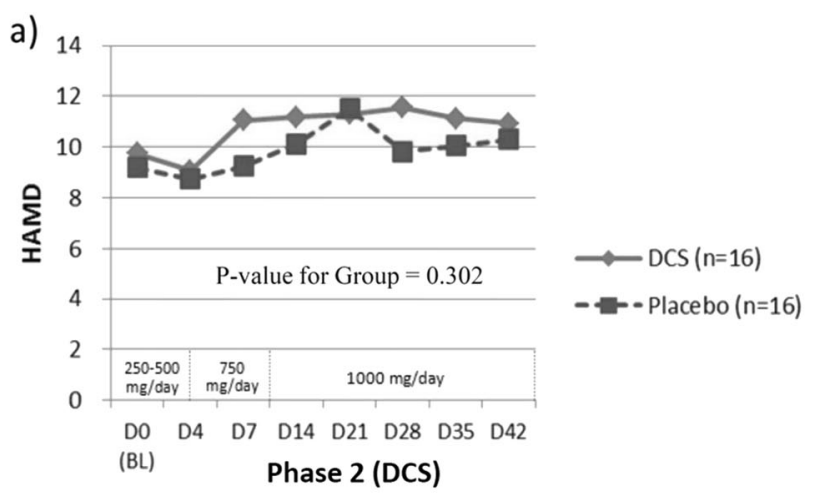

b)

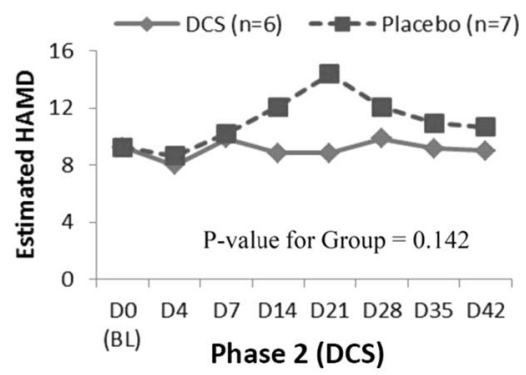

d)

DCS

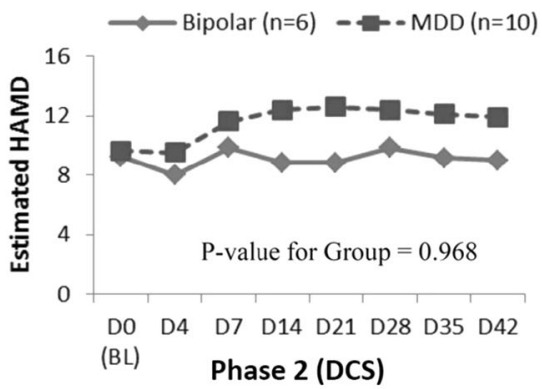

c)

MDD

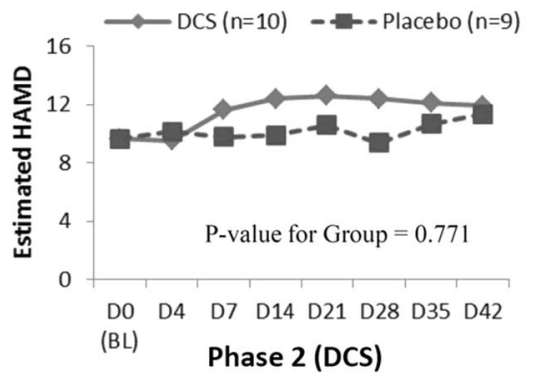

e)

Placebo

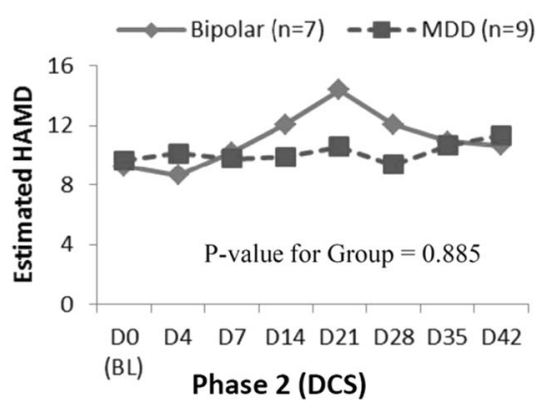

Fig. 3 Trajectory of depression in phase 2 double-blind randomized placebo control study (the mixed model with adjustment of baseline score, age, and sex). a Total patients, b bipolar disorder patients, c major depressive disorder (MDD) patients, d D-cycloserine group, and e placebo group

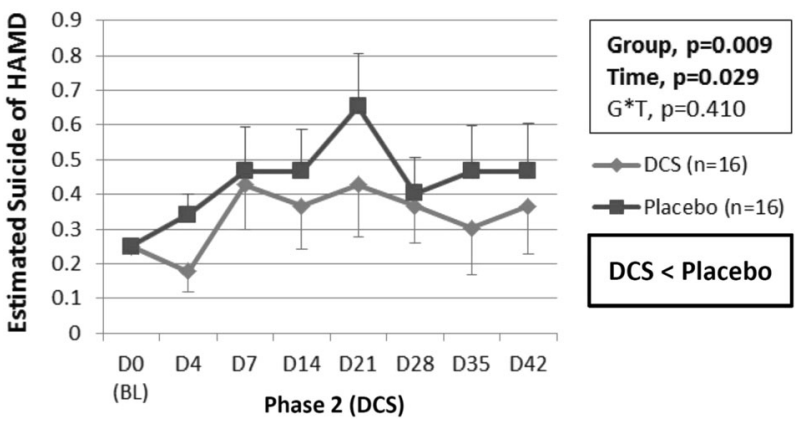

Fig. 4 Trajectory of suicide (item 3 of HAMD) in phase 2 doubleblind randomized placebo control study. HAMD Hamilton Depression Rating Scale, DCS D-cycloserine

antidepressant effect of a single ketamine infusion may last for $\sim 4$ weeks in Taiwanese patients with TRD at average baseline depression severity (baseline HAMD score $=23$ ) [7]. Murrough et al. reported that the median and 75th percentiles time to depression relapse were 18 and 27 days, respectively, after six infusions of ketamine [33]. Thus, the antidepressant effect of two ketamine infusions may last for as long as 1 month. Longer studies may be needed to unconfound the antidepressant effects of repeated ketamine exposure and DCS maintenance. Besides, the duration of treatment response to repeated ketamine infusions would still need further investigation among Caucasian and Taiwanese patients with TRD.

Another study finding was that two ketamine infusions may improve the overall treatment response (up to 65\%) in Taiwanese patients with TRD, although this inference is based on open-label data. In an open-label study of 12 patients with TRD receiving repeated ketamine infusions, Shiroma et al. reported that the cumulative response rate increased from $25 \%$ after the first ketamine infusion to $~ 91 \%$ after six infusions [36]. Murrough et al. also demonstrated a response rate of $62.5 \%$ after the first ketamine infusion with $70.8 \%$ response after a series of six thrice weekly infusions [33].

Our study had several limitations. First, our study was an add-on DCS study because the medications normally used by patients with TRD were not discontinued during the DCS augmentation treatment (Supplementary Table 2). Therefore, the potential maintaining effects of DCS could have resulted from a 
combinatory or regulatory effect of DCS with the medications that the patients were already using. However, the medications were not changed during the study period; therefore, the findings can be appropriately explained as the add-on effect of DCS. Furthermore, the add-on study design was ethically appropriate for such severely depressed patients and provided naturalistic data. Second, only 2 patients reported no suicide ideation at baseline, but 22 patients scored equal to 1 at HAMD item 3 , and 25 patients scored $\geq 2$ at HAMD item 3 in this study. However, item 3 of the HAMD rather than a rating scale specific to suicide ideation, such as the Scale for Suicide Ideation, was used as the clinical suicide covariate in the mixed model analysis; hence, we could not comprehensively investigate the potential role of DCS in the various dimensions of suicidality. Third, in addition, our current study only suggested that add-on DCS may maintain the antisuicidal effect of low-dose ketamine infusion after patients with TRD achieved the treatment response. Use of DCS may not generalize to patients with more severe symptoms of suicide. Furthermore, it appeared that the difference of HAMD item 3 may be due to a worsening in the placebo group rather than a significant improvement in the DCS group. Whether DCS may be effective for acute or severe suicide ideation would need further investigation. Fourth, there was a lack of a biomarker obtained to indicate whether DCS was having the antidepressant and antisuicidal effects in our study.

In conclusion, two ketamine infusions increase the treatment response rate, up to $65 \%$, in Taiwanese patients with treatmentresistant major depression and bipolar depression compared with a single ketamine dose. DCS augmentation may maintain the antisuicidal effect of ketamine, although it cannot maintain the overall antidepressant effect of ketamine. Repeated ketamine infusion followed by DCS augmentation treatment may be beneficial for patients with TRD who had a high treatment resistance and a suicide risk. The mechanisms underlying the potentially beneficial effect of DCS in depression and suicidality are not well understood, and need further investigation.

\section{FUNDING AND DISCLOSURE}

The study was sponsored by grants from Ministry of Science and Technology, Taiwan (101-2314-B-010-060, 102-2314-B-010-060, 107-2314-B-075-063-MY3, and 108-2314-B-075-037), Taipei Veterans General Hospital (V106B-020, V107B-010, and V107C-181), and the Kun-Po Soo Medical Foundation. Dr. Krystal was supported by the National Center for PTSD of the U.S. Department of Veterans Affairs. The funding source had no role in the any process of study. Dr Krystal acknowledges the following relevant financial interests: $\mathrm{He}$ is a co-sponsor of a patent for the intranasal administration of ketamine for the treatment of depression and suicide that was licensed by Janssen Pharmaceuticals, the maker of S-ketamine. He has a patent related to the use of riluzole to treat anxiety disorders that was licensed by Biohaven Medical Sciences. He has stock or stock options in Biohaven Medical Sciences, ARett Pharmaceuticals, Blackthorn Therapeutics, Luc Therapeutics, and Storm Pharmaceuticals. He consults broadly to the pharmaceutical industry, but his annual income over the past year did not exceed 5000 for any organization. He receives over 5000 in income from the Society of Biological Psychiatry for editing the journal Biological Psychiatry. The other author declared no conflict of interest.

\section{ACKNOWLEDGEMENTS}

We express our gratitude to all patients who kindly participated in this study. We thank all research assistants, physicians, pharmacists, and nursing staffs at D020 Unit of Taipei Veterans General Hospital for their assistance during the study process, without whom this work could not have been possible. We thank Mr I-Fan Hu for his support and friendship. The participation of Dr Krystal was supported by the US
Department of Veterans Affairs via its support for the VA National Center for PTSD, the US National Institute on Alcohol Abuse and Alcoholism (P50AA12870, M01RR00125), and the US National Center for Advancing Translational Science (UL1 RR024139). None of the aforementioned funding organizations had any role in the study design, data collection, analysis, interpretation of result, writing of the report, and the ultimate decision to submit the paper for publication.

\section{ADDITIONAL INFORMATION}

Supplementary Information accompanies this paper at (https://doi.org/10.1038/ s41386-019-0480-y).

Publisher's note: Springer Nature remains neutral with regard to jurisdictional claims in published maps and institutional affiliations.

\section{REFERENCES}

1. Howland RH. Sequenced treatment alternatives to relieve depression

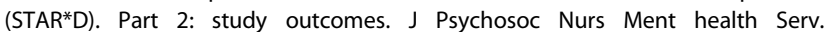
2008:46:21-4.

2. Gitlin M. Treatment-resistant bipolar disorder. Mol Psychiatry. 2006;11:227-40.

3. Schoeyen HK, Kessler U, Andreassen OA, Auestad BH, Bergsholm P, Malt UF, et al. Treatment-resistant bipolar depression: a randomized controlled trial of electroconvulsive therapy versus algorithm-based pharmacological treatment. Am J Psychiatry. 2015;172:41-51.

4. Poon SH, Sim K, Sum MY, Kuswanto CN, Baldessarini RJ. Evidence-based options for treatment-resistant adult bipolar disorder patients. Bipolar Disord. 2012;14:573-84

5. Al-Harbi KS. Treatment-resistant depression: therapeutic trends, challenges, and future directions. Patient Prefer Adherence. 2012;6:369-88.

6. Kishimoto T, Chawla JM, Hagi K, Zarate CA, Kane JM, Bauer M, et al. Single-dose infusion ketamine and non-ketamine $\mathrm{N}$-methyl-d-aspartate receptor antagonists for unipolar and bipolar depression: a meta-analysis of efficacy, safety and time trajectories. Psychol Med. 2016;46:1459-72.

7. Su TP, Chen MH, Li CT, Lin WC, Hong CJ, Gueorguieva R, et al. Dose-related effects of adjunctive ketamine in taiwanese patients with treatment-resistant depression. Neuropsychopharmacology. 2017;42:2482-92.

8. Murrough JW, losifescu DV, Chang LC, Al Jurdi RK, Green CE, Perez AM, et al. Antidepressant efficacy of ketamine in treatment-resistant major depression: a two-site randomized controlled trial. Am J Psychiatry. 2013;170:1134-42.

9. Wilkinson ST, Ballard ED, Bloch MH, Mathew SJ, Murrough JW, Feder A, et al. The effect of a single dose of intravenous ketamine on suicidal ideation: a systematic review and individual participant data meta-analysis. Am J Psychiatry. 2018;175:150-8.

10. losifescu DV. Maintaining the initial clinical response after ketamine in bipolar and unipolar depression: an important next-step challenge. J Clin Psychiatry. 2015;76:738-40.

11. Krystal JH, Sanacora G, Duman RS. Rapid-acting glutamatergic antidepressants: the path to ketamine and beyond. Biol Psychiatry. 2013;73:1133-41.

12. Heresco-Levy U, Gelfin G, Bloch B, Levin R, Edelman S, Javitt DC, et al. A randomized add-on trial of high-dose D-cycloserine for treatment-resistant depression. Int J Neuropsychopharmacol. 2013;16:501-6.

13. Henter ID, de Sousa RT, Zarate CA, Jr. Glutamatergic modulators in depression. Harv Rev Psychiatry. 2018;26:307-19. https://doi.org/10.1097/HRP.0000000000000183.

14. Heresco-Levy U, Javitt DC, Gelfin Y, Gorelik E, Bar M, Blanaru M, et al. Controlled trial of D-cycloserine adjuvant therapy for treatment-resistant major depressive disorder. J Affect Disord. 2006;93:239-43.

15. Kantrowitz JT, Halberstam B, Gangwisch J. Single-dose ketamine followed by daily D-Cycloserine in treatment-resistant bipolar depression. J Clin Psychiatry. 2015;76:737-8

16. Teng CT, Demetrio FN. Memantine may acutely improve cognition and have a mood stabilizing effect in treatment-resistant bipolar disorder. Rev Bras Psiquiatr. 2006;28:252-4.

17. Sheehan DV, Lecrubier Y, Sheehan KH, Amorim P, Janavs J, Weiller E, et al. The Mini-International Neuropsychiatric Interview (M.I.N.I.): the development and validation of a structured diagnostic psychiatric interview for DSM-IV and ICD-10. J Clin Psychiatry. 1998;59(Suppl 20):22-33. quiz 34-57.

18. van Belkum SM, Geugies H, Lysen TS, Cleare AJ, Peeters F, Penninx B, et al. Validity of the maudsley staging method in predicting treatment-resistant depression outcome using the Netherlands study of depression and anxiety. J Clin Psychiatry. 2018;79. pii: 17m11475. https://doi.org/10.4088/JCP.17m11475.

19. Hamilton M. A rating scale for depression. J Neurol Neurosurg Psychiatry. 1960;23:56-62.

20. Girgenti MJ, Ghosal S, LoPresto D, Taylor JR, Duman RS. Ketamine accelerates fear extinction via mTORC1 signaling. Neurobiol Dis. 2017;100:1-8. 
Maintenance of antidepressant and antisuicidal effects by D-cycloserine... $\mathrm{MH}$ Chen et al.

21. Danysz W, Parsons CG. Glycine and N-methyl-D-aspartate receptors: physiological significance and possible therapeutic applications. Pharm Rev. 1998;50:597-664.

22. Sheinin A, Shavit S, Benveniste M. Subunit specificity and mechanism of action of NMDA partial agonist D-cycloserine. Neuropharmacology. 2001;41:151-8.

23. Rouaud E, Billard JM. D-cycloserine facilitates synaptic plasticity but impairs glutamatergic neurotransmission in rat hippocampal slices. $\mathrm{Br} J$ Pharmacol. 2003;140:1051-6.

24. Billard JM, Rouaud E. Deficit of NMDA receptor activation in CA1 hippocampal area of aged rats is rescued by D-cycloserine. Eur J Neurosci. 2007;25:2260-8.

25. Gupta SC, Hillman BG, Prakash A, Ugale RR, Stairs DJ, Dravid SM. Effect of Dcycloserine in conjunction with fear extinction training on extracellular signalregulated kinase activation in the medial prefrontal cortex and amygdala in rat. Eur J Neurosci. 2013;37:1811-22.

26. Baker KD, McNally GP, Richardson R. d-Cycloserine facilitates fear extinction in adolescent rats and differentially affects medial and lateral prefrontal cortex activation. Prog Neuropsychopharmacol Biol Psychiatry. 2018;86:262-9.

27. Cherry KM, Lenze EJ, Lang CE. Combining d-cycloserine with motor training does not result in improved general motor learning in neurologically intact people or in people with stroke. J Neurophysiol. 2014;111:2516-24.

28. Gunthner J, Scholl J, Favaron E, Harmer $\mathrm{CJ}$, Johansen-Berg $\mathrm{H}$, Reinecke A. The NMDA receptor partial agonist $d$-cycloserine does not enhance motor learning. J Psychopharmacol. 2016;30:994-9.
29. Gujral S, Dombrovski AY, Butters M, Clark L, Reynolds CF, 3rd, Szanto K. Impaired executive function in contemplated and attempted suicide in late life. Am J Geriatric Psychiatry. 2014;22:811-19. https://doi.org/10.1016/j.jagp.2013.01.025.

30. Davis M, Ressler K, Rothbaum BO, Richardson R. Effects of D-cycloserine on extinction: translation from preclinical to clinical work. Biol Psychiatry. 2006;60:369-75.

31. Scheeringa MS, Weems CF. Randomized placebo-controlled D-cycloserine with cognitive behavior therapy for pediatric posttraumatic stress. J Child Adolesc Psychopharmacol. 2014;24:69-77.

32. Andrade C. Ketamine for depression, 4: in what dose, at what rate, by what route, for how long, and at what frequency? J Clin Psychiatry. 2017;78:e852-7.

33. Murrough JW, Perez AM, Pillemer S, Stern J, Parides MK, aan het Rot $M$, et al. Rapid and longer-term antidepressant effects of repeated ketamine infusions in treatment-resistant major depression. Biol Psychiatry. 2013;74:250-6.

34. Rasmussen KG, Lineberry TW, Galardy CW, Kung S, Lapid MI, Palmer BA, et al. Serial infusions of low-dose ketamine for major depression. J Psychopharmacol. 2013:27:444-50.

35. Phillips JL, Norris S, Talbot J, Birmingham M, Hatchard T, Ortiz A, et al. Single, repeated, and maintenance ketamine infusions for treatment-resistant depression: a randomized controlled trial. Am J Psychiatry. 2019;176:401-9.

36. Shiroma PR, Johns B, Kuskowski M, Wels J, Thuras P, Albott CS, et al. Augmentation of response and remission to serial intravenous subanesthetic ketamine in treatment resistant depression. J Affect Disord. 2014;155:123-9. 